\title{
Haploidentical Hematopoietic Stem Cell Transplantation (Haplo-SCT) with Post-Transplant Cyclophosphamide for haematological malignancies: a single cen- tre experience
}

Dmitry V. Motorin, Renat S. Badaev, Diana V. Babenetskaya, Nataliya A. Ilyina, Tatyana O. Silina, Georgiy G. Baratashvili, Vladimir V. Ivanov, Yuliya A. Alexeeva, Andrey Yu. Zaritskey

Federal Almazov North-West Medical Research Centre, Saint Petersburg, Russia.

Contact: Dr.Dmitry V.Motorin

E-mail: almazov-bmt@mail.ru

\section{Introduction}

For many patients with hematological malignancies, hematopoietic stem cells transplantation is the only potentially curative treatment option, but only $30 \%$ of patients have fully HLA-matched donor. In case of urgent need to transplant, use of alternative haploidentical donor can be the only chance for cure. For a long time, usage of haploidentical SCT was limited due to high rate of severe GVHD and graft failure. Introduction of GVHD prophylaxis with post-transplant Cyclophosphamide (PT-Cy) allowed overcome this barrier [1, 2]. Unfortunately many patients relapse after transplant. In this case, different treatment strategies can be used for induction of remission [4].

\section{Materials and methods}

Since 2014, 38 patients underwent 44 Haplo-SCTs at our centre. Six patients received second haplo-SCT because of graft failure or relapse. Five patients were transplanted from the same donor and one patient from another donor. Mean age was 38 years (21-61). Twenty two patients were males, and 16 females. The most common diagnosis was acute leukemia. Patients had AML $(\mathrm{N}=15), \mathrm{CML}(\mathrm{N}=7), \mathrm{HL}(\mathrm{N}=6)$, ALL $(\mathrm{N}=2)$, Burkitt lymphoma $(\mathrm{N}=2)$, T-lymphoblastic lymphoma $(\mathrm{N}=2)$, AL with mixed phenotype $(\mathrm{N}=1)$, NKleukemia $(\mathrm{N}=1)$, PMBCL $(\mathrm{N}=1)$ and primary myelofibrosis $(\mathrm{N}=1)$. 25 patients had relapsed or refractory disease. 15 patients proceeded to transplant without remission. Conditioning regimens were: Flu-Cy-Mel $(\mathrm{N}=22)$, Bu-FluCy $(\mathrm{N}=10)$, Flu-Cy-TBI $(\mathrm{N}=3)$, Flamsa-RIC $(\mathrm{N}=3)$, other regimens $(\mathrm{N}=6)$. PT-Cy, MMF and CsA were used for GVHD prophylaxis. Stem cell sources were either peripheral blood $(\mathrm{N}=37)$ or bone marrow $(\mathrm{N}=7)$. Mean transplant CD34+cell dose was $4.09 \mathrm{Mln} / \mathrm{kg}(0.96-12.78)$.

\section{Results}

Mean time to engraftment was 27 days for platelets and 17 for neutrophils. 3 patients $(7.8 \%)$ had primary transplant failure. Two of them had second transplantation from the same donor and one eventually engrafted. 8 patients proceeded to relapse (21\%). Among them, 6 had acute leukemia (two had molecular relapse, and four, bone marrow relapse). Six patients were treated with DLI, azacitidine +DLI (in case of acute leukemia), 4 patients had second transplantation. Different chemotherapy regimens were used in relapse. Eventually, 6 patients achieved remission. 23 patients (61\%) developed acute GVHD. 14 (37\%) patients had Grade III-IV GVHD, all of them exhibited skin form, 7 (18\%) patients had gastrointestinal form and $4(11 \%)$ had hepatic form. Mean term of GVHD onset was 38 (13-130) days. 5 patients (13.2\%) developed chronic GVHD (3 had skin damage, 1 had liver damage and 1 had muscle-skeletal form). Only 4 patients needed second-line GVHD treatment. Rate of hemorrhagic cystitis, a common complication of cyclophosphamide, was $8.9 \%(\mathrm{~N}=3)$. All cystitis episodes resolved on massive infusion therapy.

By May 2016 OS of all patients was $55 \%(\mathrm{~N}=21)$, DFS was $47 \%(\mathrm{~N}=18)$. TRM was $16 \%(\mathrm{~N}=6)$. Rate of transplantrelated mortality (at $<30$ days after transplantation) was $13 \%$ 
$(\mathrm{N}=5)$. Causes of death at late post-transplant period were as follows: infections $(\mathrm{N}=9)$, progression $(\mathrm{N}=3)$, GVHD $(\mathrm{N}=3)$, graft rejection $(\mathrm{N}=2)$.

\section{Conclusion}

Haplo-SCT is a possible alternative in patients without HLAcompatible donor. Conditioning regimen with Melphalan can be used instead of TBI-based regimens. GvHD prophylaxis with PT-Cy is feasible and effective. Different post-transplant strategies can be used for re-induction of remission in case of relapse. Disease status before transplantation has great influence on OS and PFS.

\section{References}

1. Kanakry CG, de Lima MJ, Luznik L. Alternative donor allogeneic hematopoietic cell transplantation for acute myeloid leukemia. SeminHematol. 2015;52(3):232-242.
2. Dietrich S, Finel H, Martinez C. Post-transplant cyclophosphamide-based haplo-identical transplantation as alternative to matched sibling or unrelated donor transplantation for non-Hodgkin lymphoma: a registry study by the European society for blood and marrow transplantation. Leukemia. 2016; 30(10):2086-2089.

3. Leo Luznik, Paul V. O'Donnell, J. Fuchs Ephraim. Post-transplantation cyclophosphamide for tolerance induction in HLA-haploidentical BMT. Semin Oncol .2012; 39(6):683-693

4. Amer M Zeidan, Patrick M Forde, Heather Symons. HLA-haploidentical donor lymphocyte infusions for patients with relapsed hematologic malignancies after related HLA-haploidentical bone marrow transplantation. Biol Blood Marrow Transplant. 2014; 20(3): 314-318.

\section{Keywords}

Bone marrow transplantation, haploidentical, T cell-repleted graft, cyclophosphamide, high-dose, post-transplant.

\section{| Проведение гаплоидентичной трансплантации костного мозга (гапло-ТКМ) с использованием посттрансплантационного циклофосфана: опыт одного центра}

Дмитрий В. Моторин, Ренат Ш. Бадаев, Диана В. Бабенецкая, Наталья А. Ильина, Татьяна О. Силина, Георгий Г. Бараташвили, Владимир В. Иванов, Юлия А. Алексеева, Андрей Ю. Зарицкий

Северо-Западный Федеральный Медицинский Исследовательский Центр им. В.А. Алмазова.

\section{Введение}

Аллогенная трансплантация костного мозга (алло-ТКМ) является единственной потенциальной возможностью излечения от онкогематологического заболевания. Только у $30 \%$ пациентов удается найти полностью совместимого донора. В ситуации, когда требуется неотложное проведение алло-ТКМ, выбор альтернативного гаплоидентичного донора может быть единственной возможностью. В течение длительного времени проведение гапло-ТКМ было затруднено, в связи с высоким риском развития тяжелой формы реакции трансплантат против хозяина (РТПХ), а также неприживления трансплантата. Применение метода профилактики РТПХ с использованием посттрансплантационного циклофосфана, позволяет преодолеть эти осложнения $[1,2]$. К сожалению, у большого количества пациентов наблюдается развитие рецидива после трансплантации. В этом случае могут быть применены различные методы лечения для достижения ремиссии.

\section{Материалы и методы}

Начиная с 2014 г, в нашем центре проведено 44 гапло-ТКМ у 38 пациентов. Шести пациентам была проведена повторная алло-ТКМ в связи с неприживлением трансплантата или рецидивом, из них одному пациенту проведена трансплантация со сменой донора. Средний возраст пациентов 38 лет (21-61). Двадцать два пациента - мужчины, 16 - женщины. Наиболее частым диагнозом был острый лейкоз. Заболевания были представлены: острый миелобластный лейкоз $(\mathrm{N}=15)$, хронический миелолейкоз $(\mathrm{N}=7)$, лимфома Ходжкина $(\mathrm{N}=6)$, острый лимфобластный лейкоз $(\mathrm{N}=2)$, лимфома Беркитта $(\mathrm{N}=2)$, Т-лимфобластная лимфома $(\mathrm{N}=2)$, острый лейкоз со смешанным фенотипом $(\mathrm{N}=1)$, NK-клеточный лейкоз $(\mathrm{N}=1)$, первичная медиастенальная лимфома $(\mathrm{N}=1)$, первичный миелофиброз $(\mathrm{N}=1)$. У 25 пациентов наблюдалось рецидивирующее или рефрактерного течение заболевания. Режимы кондиционирования: Flu-CyMel - 2, Bu-Flu-Cy - 10, Flu-Cy-TBI - 3, Flamsa-RIC - 3, 
другие режимы - 6. Режим профилактики РТПХ: посттрансплантационны циклофосфан, микофенолата мофетил, циклоспорин. В качестве источника стволовых клеток использовались периферическая кровь $(\mathrm{N}=37)$ и костный мозг (N=7). Среднее количество пересаженных CD34+ клеток было 4,09 млн/кг (0,96 - 12,78).

\section{Результаты}

Средний период восстановления тромбоцитов был 27 дней, нейтрофилов - 17 дней. У трех пациентов (7,8\%) наблюдалось первичное неприживление трансплантата. Двоим проведена повторная ТКМ, в результате у одного из них удалось достичь приживления. Рецидив наблюдался у 8 пациентов (21\%). Среди них было шесть пациентов с острым лейкозом (у 2 молекулярный рецидив, 4 - костно-мозговой рецидив). В качестве противорецидивной терапии использовались: инфузия донорских лимфоцитов (ИДЛ), азацитидин+ИДЛ (в случае острого лейкоза), четырём пациентам проведена повторная ТКМ, различные режимы химиотерапии. В результате, шести пациентам удалось достичь ремиссии. Острая РТПХ диагностирована у 23 пациентов (61\%), РТПХ IIIIV степени у 14 (37\%). Среди них у всех пациентов наблюдалась кожная форма, 7 (18\%) - печеночная форма, 4 (11\%) кишечная форма. Среднее время развития острой РТПХ на 38 день (13 - 130). У 5 пациентов (13,2\%) наблюдалась хроническая РТПХ (у 4 поражение кожи, 1 - поражение печени, 1 - скелетно-мышечная форма). Только 4 пациентам потребовалась терапия второй линии, в связи с рефрактерностью к ГКС. Частота возникновения геморрагического цистита, как одного из частых осложнений применения циклофосфана, была $8,9 \%(\mathrm{~N}=3)$. Все случаи цистита разрешились на фоне применения массивной инфузионной терапии. На март 2016г общая выживаемость составляет 55\% (N=21), безпрогрессивная выживаемость - 47\% (N=18). Частота ранней летальности (до 30 дней после трансплантации) - $13 \%(\mathrm{~N}=5)$. Основные причины смерти в позднем посттрансплантационном периоде: инфекционные осложнения - 9, РТПХ - 3, прогрессия - 3, неприживление трансплантата -2 .

\section{Выводы}

Гапло-ТКМ является альтернативой в случае отсутствия полностью совместимого донора. В режиме кондиционирования возможно применение мелфалана, вместо облучения всего тела. Режим с использованием посттрансплантационного циклофосфана, является доступным и эффективным методом профилактики РТПХ. В случае рецидива, могут быть использованы различные методы посттрансплантационной терапии для достижения ремиссии. Статус заболевания перед трансплантацией имеет большое влияние на общую и безрецидивную выживаемость.

\section{Литература}

1. Kanakry CG, de Lima MJ, Luznik L. Alternative donor allogeneic hematopoietic cell transplantation for acute myeloid leukemia. SeminHematol. 2015;52(3):232-242.

2. Dietrich S, Finel H, Martinez C. Post-transplant cyclophosphamide-based haplo-identical transplantation as alternative to matched sibling or unrelated donor transplantation for non-Hodgkin lymphoma: a registry study by the European society for blood and marrow transplantation. Leukemia. 2016 Oct; 30(10):2086-2089.

3. Leo Luznik, Paul V. O’Donnell, J. Fuchs Ephraim. Post-transplantation cyclophosphamide for tolerance induction in HLA-haploidentical BMT. Semin Oncol .2012 Dec; 39(6):683-93

4. Amer M Zeidan, Patrick M Forde, Heather Symons. HLA-haploidentical donor lymphocyte infusions for patients with relapsed hematologic malignancies after related HLA-haploidentical bone marrow transplantation. Biol Blood Marrow Transplant. 2014; 20(3): 314-318.

\section{Ключевые слова}

Трансплантация костного мозга, гаплоидентичная, Т-деплетированный трансплантат, циклофосфан, высокие дозы, после трансплантации 